\title{
Salinité \\ de l'Aa en amont de l'écluse de Gravelines Choix d'un emplacement de prise d'eau industrielle
}

\author{
par M. Durousseau
}

Chef de la Division Etudes et Travaux de

l'Agence de Bassin Artois-Picardie

Le démarrage, en 1974, de la zone industrielle portuaire de Dunkerque et son développement attendu, qui lamènera à couvrir tout le littoral jusqu'à Calais, entraîne un accroissement important des besoins en eau potable et industrielle. Les besoins journaliers en eau de surface et leur évolution (exprimés en consommation nette, c'est-à-dire en volume perdu par évaporation ou par évacuation à la mer, sont les suivants :

\begin{tabular}{|c|c|c|}
\hline & $\frac{1973}{-}$ & $\begin{array}{c}1990-2000 \\
-\end{array}$ \\
\hline Ecluses à la mer. & 30000 & 50000 \\
\hline Eau potable. . . . . & 10000 & 150000 \\
\hline Eau industrielle. & 60000 & 200000 \\
\hline au à usage agricole. & $\simeq 200000$ & $\simeq 200000$ \\
\hline TOTAL $\left(\mathrm{m}^{3} /\right.$ jour $)$. & 300000 & 600000 \\
\hline
\end{tabular}

Les industries existantes (Usinor, Le Borax Français, Lesieur...) sont allimentées, pour l'ensemble, par de l'eau de surface. Ces industries sont fréquemment gênées, et notamment en étiage, par la présence de sel dans les eaux qu'elles prélèvent; il n'est pas rare, en effet, de mesurer des teneurs dépassant 300 à $400 \mathrm{mg} / \mathrm{l}$ pendant plusieurs semaines consécutives. Les concentrations atteignent des valeurs très élevées - 20 à $30 \mathrm{~g} / 1$ - dans les circuits internes où le recyclage poussé est de règle en raison de la faiblesse des étiages.

Ceci nous amène à préciser la façon dont est gérée la ressource en eau qui est produite dans le bassin versant de l'Aa (fig. 1). Ce bassin couvre $1760 \mathrm{~km}^{2}$, des sources au littoral compris entre Dunkerque et Calais. Sa partie amont $\left(730 \mathrm{~km}^{2}\right)$ est géologiquement constituée par la craie, affleurant sur de grandes épaisseurs, qui constitue le réservoir régulateur des eaux de surface et celui dans lequel on puise l'eau à usage domestique. La partie aval $\left(1030 \mathrm{~km}^{2}\right)$ est une plaine de delta gagnée récemment sur la mer ( $\mathrm{X}^{\mathrm{c}}$ siècle) où se mêlent la terre ferme, l'eau douce et l'eau salée.

La ressource moyenne est de $850000 \mathrm{~m}^{3} /$ jour et la ressource en étiage sévère (décennal) est de $400000 \mathrm{~m}^{3} /$ jour; l'excédent des ressources sur les besoins actuels est faible et, avec l'accroissement de ceux-ci, le bilan sera rapidement inversé.

A l'aval de Saint-Omer, et avant de diffuser dans le delta, les eaux de l'Aa sont de qualité moyenne; les résultats analytiques sur vingt prélèvements (de 1967 à 1972 inclus) sont les suivants :

$$
\begin{aligned}
\mathrm{DCO}: & 29,0 \mathrm{mg} / \mathrm{l} ; \mathrm{Cl}-: 40,0 \mathrm{mg} / \mathrm{l} ; \mathrm{NO}_{3}-: 17,0 \mathrm{mg} / 1 \\
t_{0}: & 12,0^{\circ} \mathrm{C} ; \mathrm{O}_{2} \geqslant 5,5 \mathrm{mg} / 19 \text { fois sur } 10 .
\end{aligned}
$$

L'excédent des eaux produites dans le bassin s'évacue à la mer par des ouvrages mobiles ouverts à marée basse, fermés à marée haute, et dont les plus importants sont situés à l'extrémité des canaux navigables à Calais, Gravelines (fig. 2) et Dunkerque.

La conception de ces ouvrages, qui fonctionnent en général comme des clapets, et surtout leur entretien médiocre, sont la cause d'une introduction de sel qui est maximale en étiage lorsque les tirages à la mer doivent être réduits.

Une autre source de pollution par le sel est la présence d'une nappe salée, dont le toit affleure le fond des canaux, et qui est drainée d'autant plus intensément que la cote de l'eau dans ces canaux est basse.

C'est dans ce contexte qu'il a fallu choisir l'emplacement d'une prise d'eau de surface capable, à terme, de fournir 200000 à $300000 \mathrm{~m}^{3} /$ jour à l'abri du sel, la plus proche possible des utilisateurs, implantés le long de la côte entre Gravelines et Dunkerque.

Les volumes nécessaires ne peuvent être prélevés, en raison de leur importance, que dans un canal navigable 
dont la section, qui est supérieure à celle des canaux agricoles, autorise un tel débit. Les canaux, situés de part et d'autre de l'Aa canalisé à l'aval de Watten, ont été écartés en raison de leur cote plus basse que celle de l'Aa; ils participent, de ce fait, au drainage de la nappe salée contre laquelle il est difficile de se prémunir. En outre, le développement de la navigation, en particulier à Dunkerque, ne pouvait qu'aggràyer la pénétration saline par les écluses séparant l'eau douce de la mer et ce malgré les précautions prises, notamment à Mardyck ${ }^{a}$ ).

Dans ces conditions, il ne restait, pour implanter une prise d'eau, que l'Aa canalisé et les tronçons de canaux à la même cote qui y débouchent avec les choix suivants:

- implanter cette prise très en amont, à Watten, où le sel pénétrant à Gravelines ne remonte pas;

- ou bien la localiser plus près des utilisateurs, réduisant d'autant la longueur de l'adduction, mais la plaçant dans la zone d'influence du coin salé en provenance des portes de Gravelines.

Cette implantation, étant bien sûr la plus économique, a été adoptée après que l'on eût examiné dans quelles conditions les portes de Gravelines pouvaient être aménagées et leur fonctionnement amélioré, de façon à réduire les pénétrations d'eau salée; des campagnes de mesures ont donc été organisées dans ce but, depuis 1969, pendant les périodes de basses eaux.

\section{$*$}

On s'est attaché, pendant ces campagnes, à la mesure des débits de fuite des portes 63 et $63 \mathrm{bis}$, ouvrages qui ferment le bassin Vauban respectivement côté mer et côté Aa (voir fig. 2).

Le schéma de l'évolution des niveaux (mer, bassin, Aa) en fonction du temps, toutes portes fermées, entre deux marées hautes est celui de la figure 3 .

Soient les définitions suivantes (fig. 3):

- $S$ la surface en $\mathrm{m}^{2}$ du bassin Vauban, prise égale à $30000 \mathrm{~m}^{2}$ et supposée invariable quel que soit le niveau;

- $h_{v}$ le niveau du bassin Vauban compté à partir du niveau $h_{0}$ du bassin, all temps $t_{0}$ correspondant à la fermeture des portes 63;

- $h_{a}$ le niveau de l'Aa qui reste pratiquement constant, compté à partir du niveau $h_{0}$. Le point $\mathrm{A}$ du schéma correspondant à l'instant $t_{A}$ pendant lequel les niveaux de l'Aa et du bassin Vauban sont égaux;

- $h_{3 I}$ le niveau de la mer compté à partir du niveau $h_{0}$;

- $Q_{a 1}$ le débit de fuite qui passe par les portes 63 bis dans le sens bassin Vauban vers $\mathrm{Aa}$; il a été admis que $Q_{a 1}$ pouvait être calculé comme le débit d'un orifice noyé :

$$
Q_{a 1}=k_{1}^{\prime} \sqrt{h_{v}-\overline{h_{a}}}
$$

$k_{1}^{\prime}$ étant le «coefficient de fuite » des portes 63 bis dans le sens bassin Vauban vers $\mathrm{Aa}$ (en $\mathrm{m}^{\overline{5} / 2 / \mathrm{s}}$ );

- $Q_{a 2}$ le débit de fuite qui passe par les portes 63 bis dans

(a) Voir l'article sur l'écluse de Mardyck dans le $\mathrm{n}^{\circ}$ 1/1965 de La Houille Blanche. le sens Aa vers bassin Vauban; de la même manière il a été admis que :

$$
Q_{a 2}=k_{2}^{\prime} \sqrt{h_{a}-h_{v}}
$$

$k_{2}^{\prime}$ étant le «coefficient de fuite» des portes $63 \mathrm{bis}$ dans le sens Aa vers bassin Vauban $\left(\right.$ en $\mathrm{m}^{5 / 2} / \mathrm{s}$ );

Les coefficients de fuite de l'ouvrage 63 bis ont été distingués selon l'écoulement: $k_{1}^{\prime}$ dans le sens bassin Vauban vers Aa et $k_{2}^{\prime}$ dans le sens Aa vers Bassin Vauban car, selon le sens de l'écoulement, ce ne sont pas les mêmes paires de portes qui assurent l'étanchéité.

- $Q_{v}$ est le débit de fuite qui passe par les portes $63 \mathrm{du}$ bassin vers la mer:

$$
Q_{v}=k \sqrt{h_{v}-} \overline{h_{\mathrm{II}}}
$$

$k$ étant le «coefficient de fuite» des portes 63 (en $\mathrm{m}^{5 / 2} / \mathrm{s}$ ) dans le sens bassin Vauban vers la mer.

Pendant la période $t_{0}-t_{A}$, le bassin Vauban s'est vidé sous l'effet des fuites aux portes 63 et 63 bis et l'on peut exprimer ce volume de vidange $V_{1}$ par :

$V_{1}=S . h_{A}=k \int_{t_{0}}^{t_{A}}\left(h_{v}-h_{11}\right)^{1 / 2} d t+k_{1}^{\prime} \int_{t_{0}}^{t}\left(h_{v}-h_{a}\right)^{1 / 2} d t$ soit :

$$
V_{1}=k \alpha_{1}+k_{1}^{\prime} \beta_{1}
$$

Pendant la période $t_{A}-t_{B}$, le bassin Vauban s'est vidé sous l'effet des fuites aux portes 63 , partiellement compensées par les apports des portes 63 bis de l'Aa vers le bassin; ce volume de vidange $V_{2}$ peut être exprimé par:

$$
\begin{aligned}
V_{2} & =S\left(h_{B}-h_{A}\right) \\
& =k \int_{i_{1}}^{t_{B}}\left(h_{v}-h_{M}\right)^{1 / 2} d t-k_{2}^{\prime} \int_{t_{A}}^{t_{B}}\left(h_{a}-h_{v}\right)^{1 / 2} d t
\end{aligned}
$$

soit :

$$
V_{2}=k \alpha_{2}-k_{2}^{\prime} \beta_{2}
$$

A chaque intervalle de temps $d t$, le bassin $T$ auban se vide de la quantité $\Delta h_{v}$; l'équation (2) peut s'inscrire de la façon suivante :

$$
S . \Delta h_{v}=k . d t\left(h_{v}-h_{M}\right)^{1 / 2}-k_{2}^{\prime} \cdot d t\left(h_{a}-h_{v}\right)^{1 / 2}
$$

soit :

$$
\Delta h_{v}=\left[k \cdot d t\left(h_{v}-h_{M}\right)^{1 / 2} / S\right]-\left[k_{2}^{\prime} \cdot d t\left(h_{l}-h_{v}\right)^{1 / 2 / S}\right]
$$

On arrive ainsi par approximations successives à adapter des valeurs $k$ et $k_{2}^{\prime}$ qui reconstituent la courbe de vidange réelle. Par suite, une fois connue la valeur de $k$, on en déduit à l'aide de la relation (1) ci-dessus la valeur de $k_{1}^{\prime}$ que l'on ajuste également par la même méthode (fig. 4).

\section{Commentaires et résultats}

1) On a admis que $k, k_{1}^{\prime}$ et $k_{2}^{\prime}$ étaient invariants au cours d'une descente du niveau du bassin Vauban et du niveau de la mer et qu'ils ne variaient pas au fil des marées. 


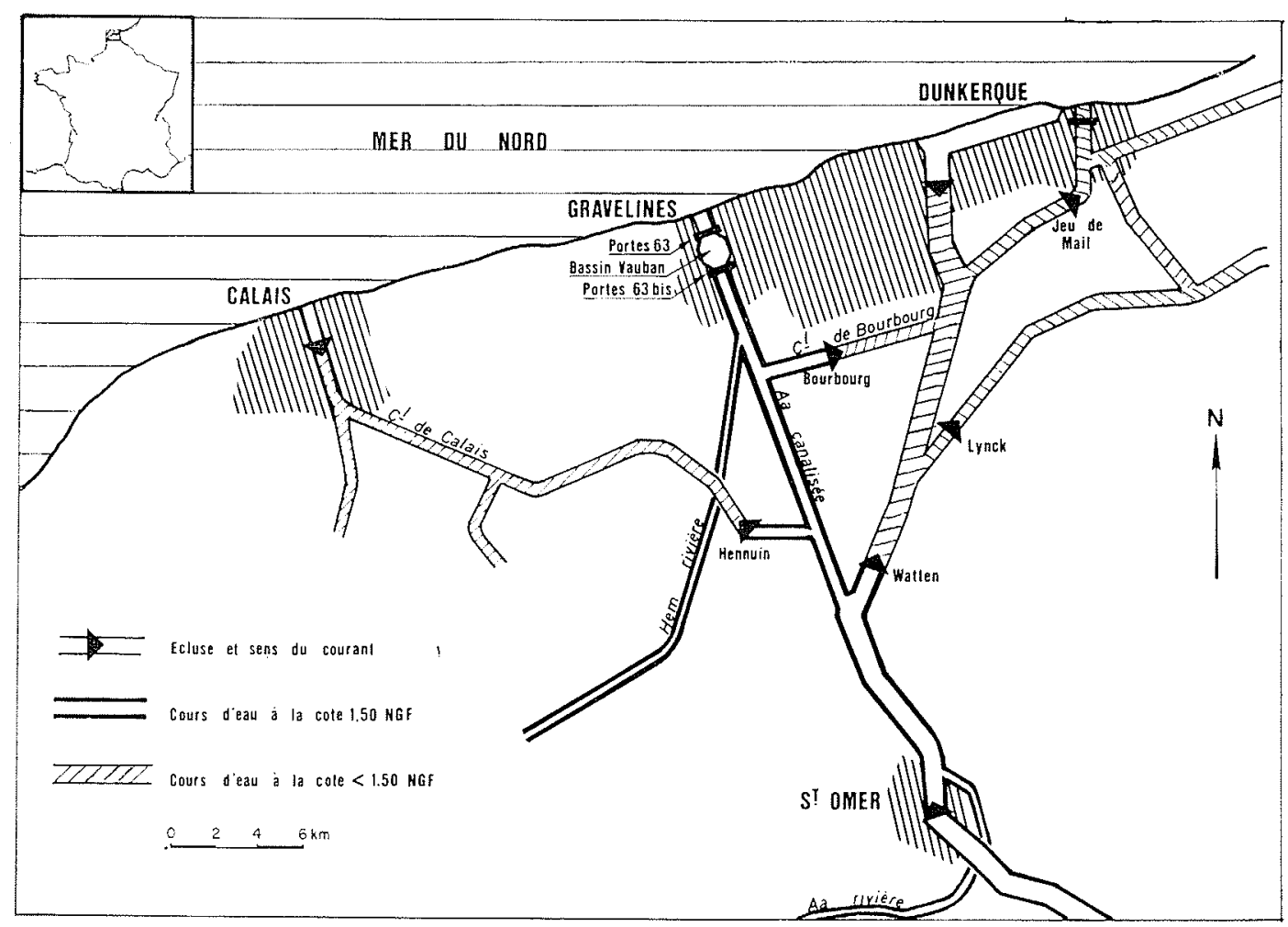

1/ Plan de situation

schéma synoptique de l'ècoulement des eaux à GRAVELINES

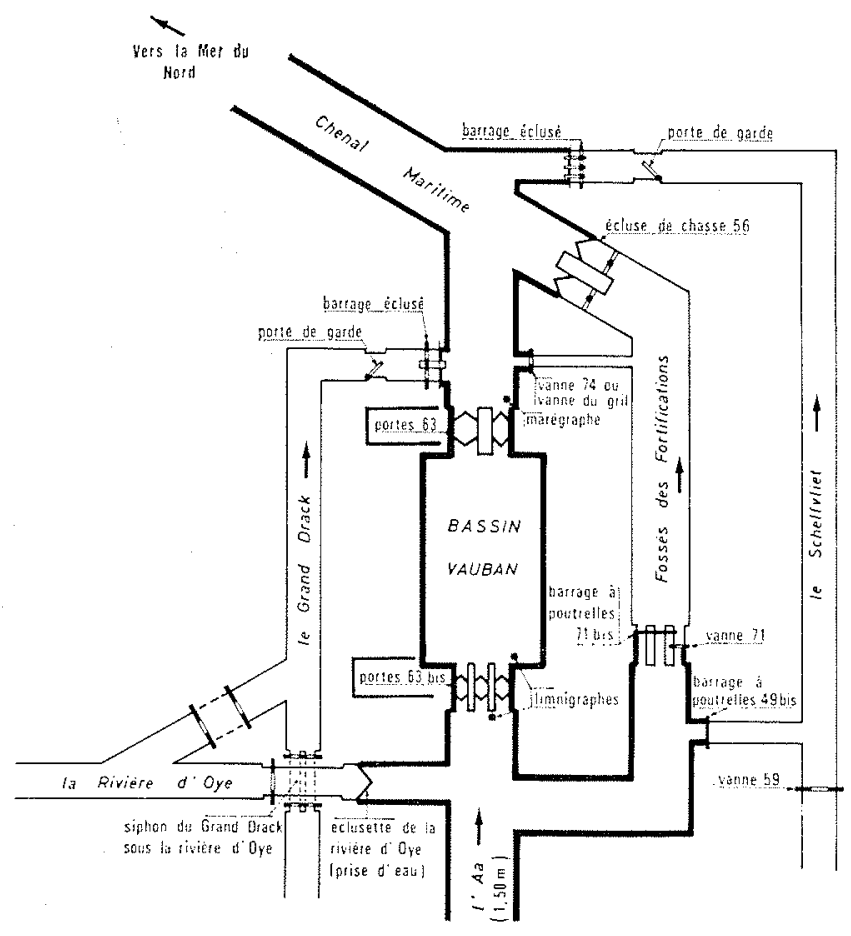

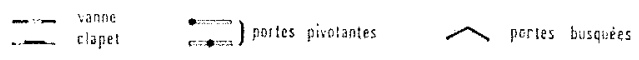

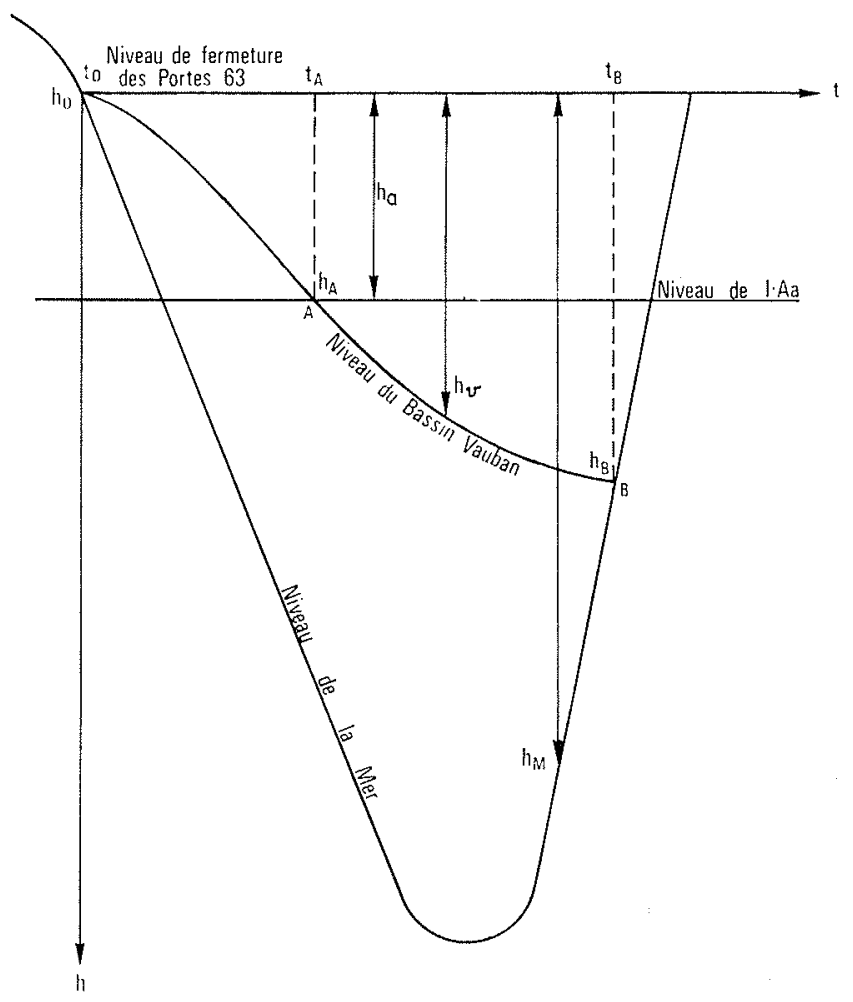

3/ 


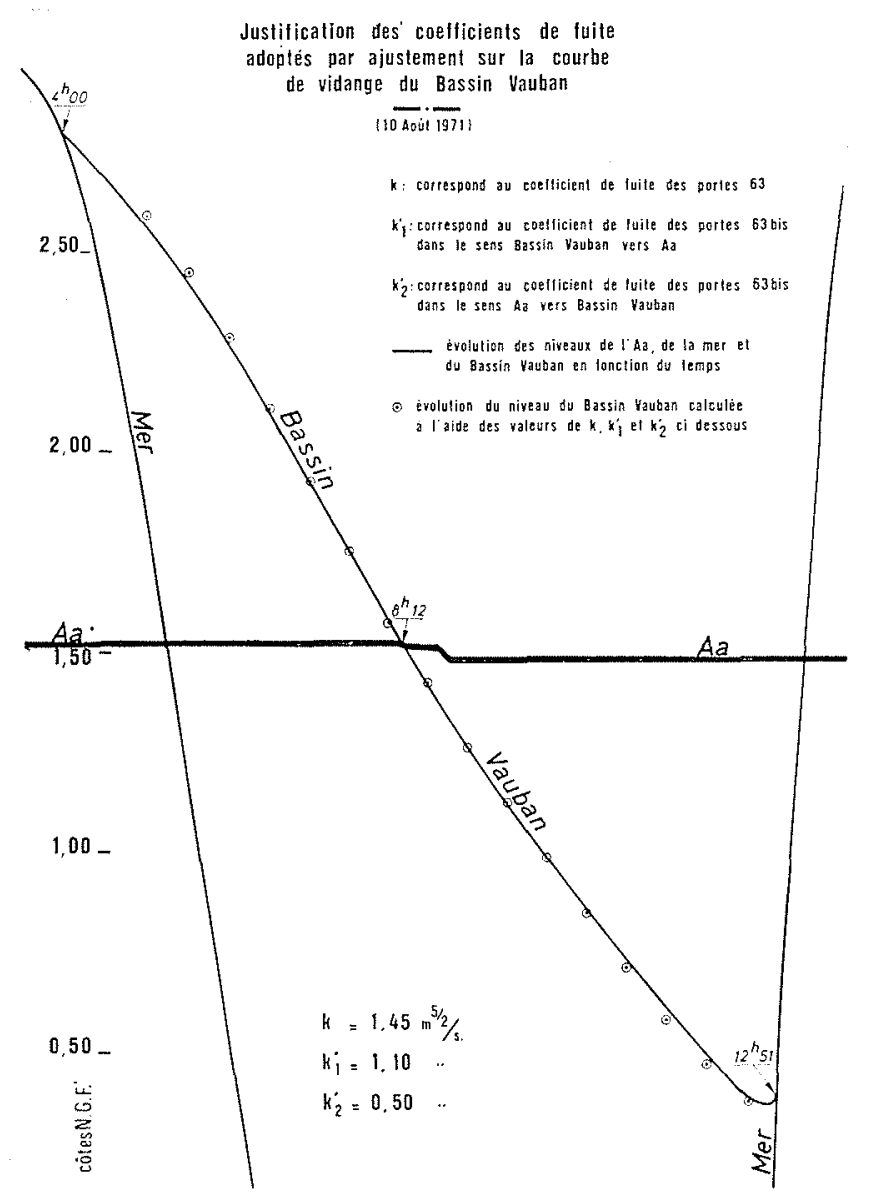

$4 /$
2) On a admis également, dans ce calcul particulier, que les densités d'eau douce et d'eau de mer étaient égales.

3) Enfin, les quantités $V_{1}$ et $V_{2}$ ne sont pas tout à fait proportionnelles à la baisse du niveau du bassin Vauban et, faute de renseignements sur la forme réelle du bassin, il est difficile d'apprécier l'erreur qu'entraîne un tel calcul du volume.

Malgré cette série d'approximations, les relations (1) et (2) ont donné pour $k / k^{\prime}$ et $k / k_{2}^{\prime}$ des résultats très voisins quelles que soient les marées et leur amplitude; l'ajustement graphique a permis ensuite de calculer les valeurs absolues de ces coefficients qui sont les suivants (avant réparation des portes) :

$$
k=1,45 \mathrm{~m}^{5 / 2} / \mathrm{s} ; k_{1}^{\prime}=1,1 \mathrm{~m}^{5 / 2} / \mathrm{s} ; k_{2}^{\prime}=0,5 \mathrm{~m}^{5 / 2} / \mathrm{s}
$$

- Les fuites de l'Aa vers le bassin Vauban (proportionnelles à $k_{2}^{\prime}$ ) sont tolérables: elles représentent en moyenne $10000 \mathrm{~m}^{3}$ par marée soit $20000 \mathrm{~m}^{3} /$ jour.

- Par contre, les fuites du bassin Vauban vers 1'Aa (coefficient $k_{1}^{\prime}$ ) sont plus importantes : $40000 \mathrm{~m}^{3} /$ jour et, en outre, sont responsables de la pénétration du sel.

- Les fuites des portes 63 sont encore plus importantes, puisqu'elles atteignent $100000 \mathrm{~m}^{3} /$ jour soit le quart de la ressource en étiage.

Parallèlement à ces mesures de débit, étaient effectuées des mesures de salinité le long de l'Aa; on a reporté à titre d'exemple, sur la figure 5, l'évolution du coin salé sur trois jours; il n'a pas pu malheureusement être mis en évidence une relation entre la longueur du coin salé, les tonnages de sel et les volumes introduits par les fuites.
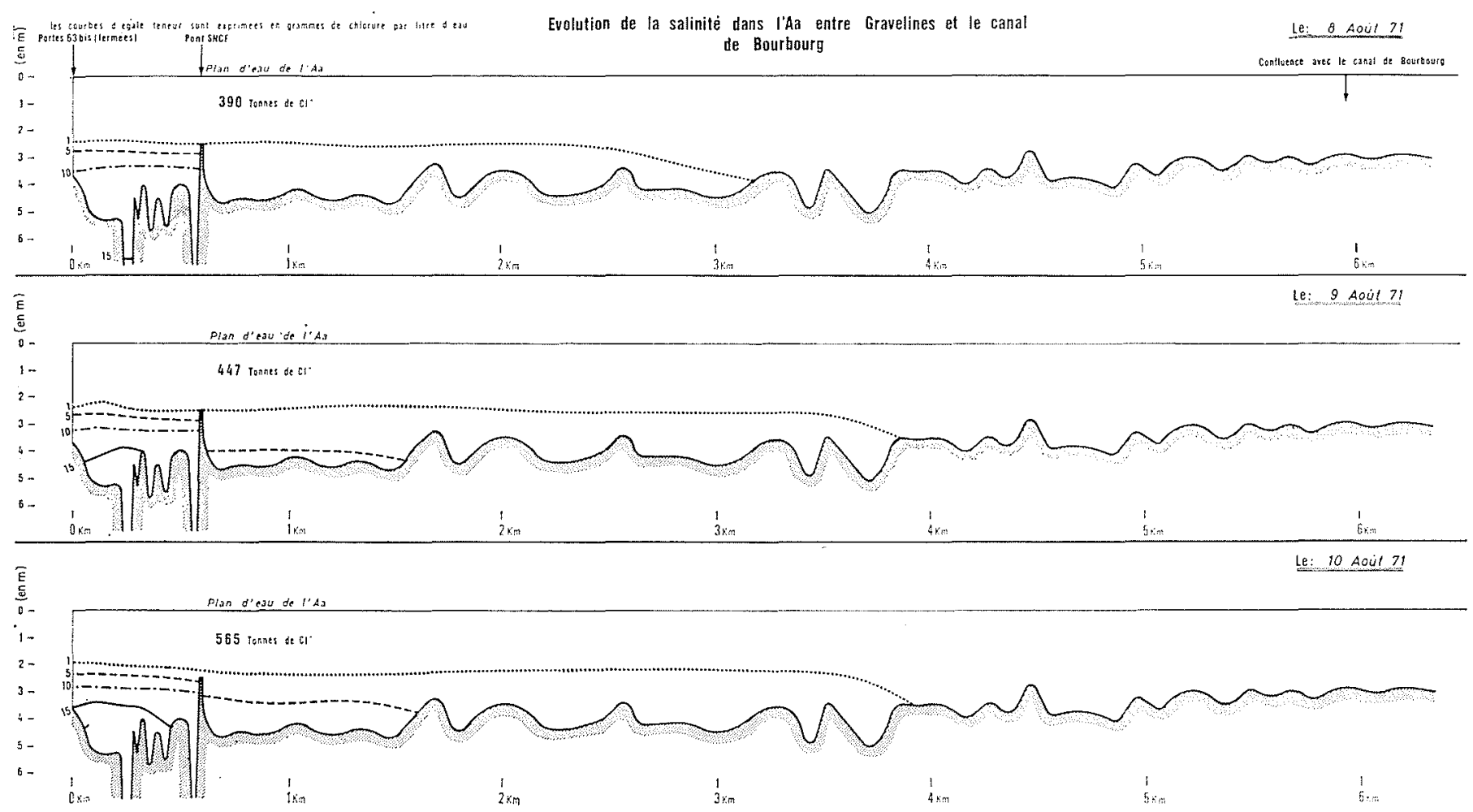
*:

En conclusion des campagnes effectuées, il a été décidé :

$1^{\circ}$ de réparer les portes 63 et 63 bis et en outre de compléter la fermeture de deux des trois passes de ce dernier ouvrage par des batardeaux métalliques à mettre en place pendant les étiages;

$2^{\circ}$ de modifier les consignes de fonctionnement des portes 63 bis, à savoir de les maintenir fermées en perma- nence (à l'exception bien sûr du temps de passage des péniches au demeurant peu nombreuses, 1 à 2 par mois);

$3^{\circ}$ d'installer une station de pompage destinée à refouler, vers le bassin Vauban, l'eau salée introduite dans l'Aa par les fuites résiduelles.

Le débit d'équipement de cet ouvrage est en cours de calcul, après vérification de l'efficacité de la remise en état des portes achevée en juin dernier.

\section{Discussion}

Président : M. C. LEFrou

M. le Président remercie M. Durousseau de son exposé plein d'enseignements et ouvre la discussion.

Pourriez-vous, demande M. G. de Marsily, nous expliquer comment il est possible de pomper dans l'Aa l'eau provenant des fuites de l'écluse du bassin Vauban, sans pomper đu même coup l'eau douce en provenance de l'amont?

Cela résulte, dit $M$. Durousseau, d'une disposition particulière des lieux. Le fond de l'Aa présente, quelques dizaines de mètres en amont des portes du bassin Vauban, une fosse profonde dont l'origine est inconnue; elle date peut-être de la guerre et pourrait être due à une bombe tombée en ce lieu. Le tirant d'eau normal est de l'ordre de $3 \mathrm{~m}$ et, par contre, cette fosse à une profondeur de $11 \mathrm{~m}$ et ne s'est pas comblée. L'eal salée, ayant de progresser vers l'amont, commence par remplir cette fosse; on peut la pomper facilement dans celle-ci et la rejeter par-dessus les portes. Grâce a un contrôle de la conductivité électrique de l'eau au droit d'un seuil naturel encore plus en amont, les pompes sont mises en routes et arrêtées automatiquement aux moments opportuns.

Aucun auditeur ne demandant la parole, M. le Président clôt à la fois la discussion et la séance, en remerciant tous ceux qui ont contribué à l'animer. 